\title{
Mathematical Analysis for Radiometric Calorimetry of a Radiating Sphere
}

\author{
L. A. Schmid* \\ National Bureau of Standards, Washington, DC 20234
}

September 1S, 1982

\begin{abstract}
Equatious are derived from which the temperature dependence of both the specific heat and the thermal diffnsivity of a spherical sample of material can be calculated from observations of the time dependence of the surface temperature and the time-rate of energy loss from the sample as it cools. The derivation takes into account the nonuniformity of the interior temperature field of the sample, and the resnlting eqnations can be applied not only to radiative cooling, but also to any other cooling mechanism that does not violate the assumed spherical symmetry. The analysis excludes change of phase, but it does take thermal expansion into account. To permit the making of estimates neeessary for the design of radiative cooling experiments, a universal temperature-time cooling curve is derived for the post-transient cooling regime of a radiating sphere of any size with arbitrary, but constant, thermal parameters.
\end{abstract}

Key words: calorimetry; Fourier equation; radiative eooling; specific heat; thermal diffusivity.

\section{Introduction}

The analysis presented in this paper is an outgrowth of a proposal made by J. H. Colwell $[1,2]$ to determine the high-temperature values of the specific heat, thermal diffusivity, and total hemispherical emissivity of a spherical sample of refractory material by making independent optical observations of the surface temperature of the sample and its timc-rate of energy loss as it cools by free radiation into a cold vacuum. The original proposal was made in the context of an experiment to be conducted on board the space shuttle, and envisaged induction heating of the sample. With this mode of heating, the total heat content of the spherical sample and its interior temperature field at the start of the observational run would be unknown. However, after an interval on the order of the characteristic thermal decay time of the sample, the interior temperature field would settle into the "post-transient regime" in which the interior field would be entirely determined by the time-dependence of the surface-temperature. Thus, in the post-transient regime it should, in principle, be possible to determine the temperaturc dependence of the thermal parameters from

"Center for Chemical Engineering, National Engineering Laboratory a knowledge of the time dependenee of the surface temperature and the rate of energy loss. The determination of the emissivity is a trivial matter, since it is proportional to the rate of energy loss divided by the fourth power of the surface temperature.

Stated in mathematical terms, the cooling sample could be regarded as mapping the temperaturedependent specific heat and diffusivity over into the time-dependent surface temperature and energy loss functions, and the analytical problem then consists of inverting this mapping so as to be able to express the two unknown thermal parameters in terms of the two obscrved time functions. The first step in carrying out this inversion, the results of which are summarized in section 2 , is to find the "surface-driven solution" of the Fourier equation for specified temperature-dependent specific heat and thermal diffusivity. This solution is completely specified by the time dependence of the surface temperature, and the time rate of change of the total heat eontent can be calculated from it. If this calculated rate of change is then equated to the fourth power of the surfaee temperature in aeeordance with the StefanBoltzmann radiation law, a nonlinear ordinary differential equation (of infinite order) results whieh ean be iteratively solved (in truncated form) for the ease of con- 
stant thermal parameters to yield a universal temperature versus time dependence for the posttransient regime. This solution, which is presented in section 3 , is useful for making the various cstimates that are necessary for the design of a radiative-cooling calorimetry experiment. At the end of section 3 an integral equation is given that could also be used as the basis of an iterative solution of the post-transient predictive problem.

In section 4 the surface-driven solution that is summarized in section 2 is inverted so as to yield expressions for specific heat and diffusivity in terms of the observed time-dependent surface temperature and time-rate of energy loss. These expressions constitute the desired solution of the calorimetric problem. The thermal parameters are expressed both in terms of truncated expansions whose coefficients involve higher-order time derivatives of the observed functions, as well as in terms of integral expressions involving retrospective weighted averages of the observed time-dependent functions. The truncated expansions, which are easier to apply than the integral expressions, ought to suffice for analyzing most post-transient experiments. In fact, in many experiments the simple approximate expressions given in eqs (44) and (45) will be sufficiently accurate. In section 4 an estimate of the range of validity of these simple expressions, as well as the range of validity of the more accurate truncated expansions, is given in terms of the magnitude of a suitably scaled dimensionless temperature. The scaling factor, which is introduced in section 3 , takes the material parameters and sphere size into account. When these parameters have values for which the truncated expansions are not accurate, then the integral expressions for the thermal parameters can be used as a basis for an iterative solution of the calorimetric problem. These integral expressions could also be used to analyze a calorimetry experiment conducted iu the transient regime, assuming that the knowledge of the surface temperature of the sample includes an interval lon the order of the characteristic decay time) that precedes the commencement of the cooling observations. For example, if a sample were held in a constant-tcmperature oven (of known temperature) long enough to become isothermal, and then suddenly removed to commence cooling which was observed for a time interval on the order of the characteristic thermal decay time, the integral expressions for the thermal parameters could be used to analyze the data.

Although the analysis of this paper was carried out with radiative cooling in mind, only in the solution of the predictive problem in section 3 is the radiative cooling law invoked. In the analysis of the calorimetric problem, the cooling law is never specified. All that is assumed is that the time dependence of the time-rate of total energy loss by the sample is known (as is the time dependence of the surface temperature).

The most obvious limitation of the analysis of this paper (aside from its restriction to spherical symmetry) is the exelusion of the possibility of phase change. That is, the spherical sample is assumed to be either entirely solid or entirely liquid throughout the experiment. In addition to this limitation, the analysis incorporates two approximations, the more significant being the neglect of the spatial variation of the diffusivity in the interior of the spherical sample. That is, the diffusivity is assumed to be a function of the surface temperature (which is a function only of time) rather than a function of the interior temperature (which is a function of the radial coordinate as well as of time). It is shown in section 2 that this approximation amounts to neglecting a very small term in the Fourier equation that has the form of an effective heat source, but, as explained in section 5 , this effective heat source can be taken into account (if necessary) by a simple iterative procedure. 'The other approximation, whose effect is completely negligible, is the neglect of the spatial variation of the mass density of the sample. That is, the overall change in average density with temperature is taken into account, but at each instant the density throughout the sample is assumed to be spatially constant. In other words, as in the case of diffusivity, the density throughout the sample is assumed to be a function of the surface temperature rather than of the interior temperature.

The literature relevant to predictive solutions of the Fourier equation is old, vast, and still growing [3,4]. However, this literature is almost exclusively devoted to the initial-value approach to the problem which requires that at some instant the interior temperature field must have some exactly specified form (most commonly, a given uniform temperature). This point of view, however, is physically inappropriate to the calorimetric problem becausc what is usually known is the history of the environment to which the sample has been exposed (i.e., the history of its surface temperature), and not the interior temperature field at any instant. It is true that, if the sample is kept in a constant-temperature oven long enough, its interior temperature will indeed be spatially uniform, but this is a special case. It would be physically more natural to replace the initial specification of the interior temperature field with the specification of the surface temperature history back to $t=-\infty$. (It is shown in section 2 that as a practical matter it is only necessary to know the surface temperature during a very short period of the past.) There is a well-known solution to the Fourier equation (cf. for example p. 247 of Ref. [3]), which has the form of a convolution of the surface 
temperature with the well-known diffusion kernel, but this solution is inappropriate to the calorimetry problem because it has a singularity at the center, and so (in the absence of a point heat source) can only be used to deseribe the temperature field in an infinite medium surrounding a spherical cavity.

The mathematical literature that is directly relevant to the determination of the thermal parameters from the observed surface temperature [5-13] unfortunately has remained within the framework of the initial-value approach. Because an arbitrarily specified timedependence for the surface temperature is generally inconsistent with a previously specified initial interior temperature field, the problem is over-specified, and certain compatibility conditions must be satisfied before the problem is well-posed. The derivation of these conditions has been an important theme in this literature. (The whole question of compatibility becomes irrelevant, of course, when the surface-driven solution is used as the basis of the analysis.) The thermal parameters have been expressed most commonly as the solution of an integral equation, but the most general case considercd so far has allowed only one of the two parameters to be an unknown function of temperature, the other being an unknown constant. Because these solutions are very different in form from the expressions given in this paper, and because the geometry considered was planar (either slab or semi-infinite medium) rather than spherical, no attempt has been made to compare the results of this paper with the previously derived expressions for the thermal parameters.

\subsection{Notation}

The main analysis involves dimensionless quantities, which are designated by bare letters, whereas the corresponding dimensional quantities are indicated by an asterisk. Time-independent unit quantities (also dimensional) are indicated by a caret. The relations existing among the three types of quantities are given in table $\mathrm{I}$ which also serves to define most of the notation. (A few more symbols will be introduced as needed.) Table I also shows how the various dimensional quantities depend on the radius of the sphere. Because of thermal expansion, both the dimensional radius $R^{*}$ and the dimensionless radius $R$ are variable, but the unit radius $\hat{R}$ is an arbitrarily chosen constant. The $R$-dependence of the various dimensionless quantities has been defined in

Table I. Basic Notation for Calorimetric Problem.

\begin{tabular}{|c|c|c|c|}
\hline Quantity & $\begin{array}{l}\text { Dimensionless } \\
\text { Symbol }\end{array}$ & $\begin{array}{l}\text { Dimensional } \\
\text { Symbol }\end{array}$ & Remarks \\
\hline $\begin{array}{l}\text { Radius of sphere } \\
\text { Radial distance } \\
\text { Gradient operator } \\
\text { Mass density }\end{array}$ & $\begin{array}{l}R(t) \\
r \\
\nabla \\
e(t)\end{array}$ & $\begin{array}{l}R^{*}=R \hat{R} \\
r^{*}=r R \hat{R} \\
\nabla^{*}=R \hat{R} \nabla \\
e^{*}=\ell \hat{\ell} / R^{3}\end{array}$ & $\begin{array}{l}0 \leqslant r \leqslant 1 \\
\left\{\begin{array}{l}Q \stackrel{-}{=} \text { constant } \\
\nabla^{*} Q^{*} \doteq 0\end{array}\right.\end{array}$ \\
\hline $\begin{array}{l}\text { Specific heat } \\
\text { Thermal conductivity } \\
\text { Thermal diffusivity } \\
\text { Total hemispherical } \\
\text { emissivity }\end{array}$ & $\begin{array}{l}c(\theta) \\
k(\theta) \\
a(\theta) \\
\varepsilon(T)\end{array}$ & $\begin{array}{l}c^{*}=c \hat{c} \\
k^{*}=(\mathbf{k} / \mathbf{R}) \hat{\mathbf{k}} \\
a^{*}=R^{2} a \hat{a} \\
\varepsilon^{*}=\varepsilon \hat{\varepsilon}\end{array}$ & $\left\{\begin{array}{l}\mathrm{k}^{*}=\mathrm{e}^{*} \alpha^{*} c^{*} \\
\hat{k}=\hat{\mathrm{Q}} \hat{\alpha} \hat{c} \\
k=\hat{Q} \alpha c\end{array}\right.$ \\
\hline $\begin{array}{l}\text { Linear time } \\
\text { Nonlinear time } \\
\text { Surface temperature }\end{array}$ & $\begin{array}{l}t \\
T(t) \\
T(t)\end{array}$ & $\begin{array}{l}t^{*}=t \hat{t} \\
d \tau=\alpha(T) d t=\alpha^{*} d t^{*} / R^{* 2} \\
T^{*}=T \hat{T}\end{array}$ & $\hat{t} \equiv \hat{R}^{2} / \hat{\alpha}$ \\
\hline $\begin{array}{l}\text { Interior temperature } \\
\text { Surface specific enthalpy } \\
\text { Interior specific }\end{array}$ & $\begin{array}{l}\Theta(t, r) \\
h(t)\end{array}$ & $\begin{array}{l}\theta^{*}=\theta \hat{T} \\
h^{*}=h \hat{h}\end{array}$ & $\begin{array}{l}(\theta)_{r=1}=T \\
\hat{h} \equiv \hat{c} \hat{T}\end{array}$ \\
\hline $\begin{array}{l}\text { enthalpy } \\
\text { Total mass } \\
\text { Total enthalpy } \\
\text { (average specific } \\
\text { enthalpy) }\end{array}$ & $\begin{array}{l}\eta(t, r) \\
H\end{array}$ & $\begin{array}{l}\eta^{*}=\eta \hat{h} \\
M^{*}=\frac{4 \pi}{3} \mathrm{R}^{* 3} Q^{*}=\left(\frac{4 \pi}{3} \hat{R}^{3} \hat{Q}\right)_{Q}=\hat{M} Q \\
H^{*}=M^{*} \eta_{\Delta v} \hat{h}=(\hat{M} \hat{C} \hat{T})_{Q} H\end{array}$ & $\begin{array}{l}(\eta)_{r=1}=h \\
H \equiv \eta_{a v}\end{array}$ \\
\hline Indep & & & \\
\hline
\end{tabular}


such a way that the dimensionless Fourier equation is completely independent of the effects of thermal expansion. (Cf. Sec. 2.) During an observational run, $R *\left(t^{*}\right)$ can be measured optically along with $T *\left(t^{*}\right)$, and then $R\left(T^{*}\right)$ can be calculated. When, for example, this $R\left(T^{*}\right)$ is entered into the expression given in table I for $\alpha^{*}$, the contribution to the $T^{*}$-dependence of $a^{*}$ that results from thermal expansion is automatically taken into account. Tliroughout the paper, except for section 3 that deals with the predictive problem, it will be assumed that $R^{*}\left(t^{*}\right), T^{*}\left(t^{*}\right)$, and $d H^{*} / d t^{*}$ are given functions of $t^{*}$ resulting from the experimental observations. From these the dimensionless functions $R(t), T(t)$, and $\dot{H}(t) \equiv$ $d H / d t$ can be directly calculated, so it will be assumcd that these too are given functions. An overhead dot will indicate differentiation with respect to the dimensionless linear time t. It should usually be possible to choose the unit time $\hat{\boldsymbol{t}}$ to be a convenient multiple of some experimentally defincd time interval, such as the interval between ohservational rcadings. Diffcrentiation with respect to the nonlinear time coordinate $\tau$ will be designated as follows: $d h / d \tau \equiv H^{(1)}$. Although it will often be desirable to choose $\hat{\varrho}, \hat{a}$, and $\hat{c}$ to be close to the values of $e^{*}, a^{*}$, and $c^{*}$ at $T^{*}=\hat{T}$ (which means that the corresponding dimensionless quantities will be close to unity at the reference temperature $\hat{T}$, this is not necessary.

\section{Surface-Driven Solution}

If the spherical sample is imagined to be immersed in a hcat reservoir of variable temperature, then changes in the interior temperature field are driven by the prescribed changes in the surface tcmperature. Assuming the absence of any interior heat sources, it follows that the interior temperature field is uniquely determined by the past history of the surface temperature up to the present moment. In mathematical terms, this corresponds to the "particular" or "driven" solution of the Fourier equation, with the surface temperature playing the role of the "driving function." This is not the most general solution, because it does not include the homogeneous solution which describes the decay of an arbitrarily specified initial interior temperature field. It is well known that the most slowly decaying term in the homogeneous solution has a time dependence proportional to $\exp \left(-\pi^{2} t\right)$ where $t$ is the dimensionless time measured in the natural time unit defined in table $I$. Neglecting the homogeneous solution amounts to assuming that the interior temperature field has been subjected to no influences other than its external environment for a period of time $t$ that is long enough so that $\exp \left(-\pi^{2} t\right)<<1$.
The time-rate at which the sample exchanges energy with its surroundings is determined by the history of the surface temperature up to the present moment. In fact, it is just equal to the time derivative of the total interior enthalpy of the sample. Thus, once the time history of the surface temperature has been specified, the time-rate of energy loss or gain of the sample is completely determined. The analysis of this section leads to expressions (summarized in tables $\mathrm{V} \& \mathrm{VI}$ ) relating tlie time-rate of total energy change of the sample to the surface temperature for more exactly, the specific enthalpy at the surface), and these expressions suffice for the analysis of both the predictive and the calorimetric problems.

The dimensional Fourier equation is given in the two forms ( $\mathrm{l}$ a) and ( $1 \mathrm{~b}$ ) of table II, the only difference being the representation of the part of the heat flux that results from radial motion caused by thermal expansion or contraction. In eq (la) it is represented in terms of the material velocity $v^{*}$ at a point $r^{*}$ that is fixed in the laboratory (inertial) frame, whereas in eq (1b) the motion is taken into account by the fact that the time derivative is taken with respect to fixed $r$ rather than fixed $r^{*}$, where $r$ is the dimensionless radial vector that is attached to a particular material particle and moves with it. Although $\mathrm{v}^{*}$, whieh is the material velocity associated with thermal expansion or contraetion, is negligibly small, the point to be made is that the right-hand side of the dimensionless Fourier equation given in eq (2) is rigorously correet, and the fact that the time derivative is taken at constant $r$ rather than $r^{*}$ does not represent an approximation.

In eq (3) the internal enthalpy density $\eta$ is introduced in order to replace the internal temperature $\theta$. This replacement is doubly advantageous: First, a comparison of eqs (2) and (4) shows that it reduces the number of thermal parameters that appear in the equation. Second, the enthalpy density is really the quantity of physieal interest, because the objeetive of the analysis is to integrate it over the volume of the sample in order to arrive at an expression for the time-rate of change of the total enthalpy (heat content) of the sample.

Equation (4a) still contains the temperaturedependent diffusivity $a(\Theta)$, and this fact not only complicates the equation, but also prevents it from being universal in the sense of having the same form regardless of the material properties of the sample. If the diffusivity were a funetion only of $t$ and not of $r$, it could be eliminated from the equation by replacing the linear dimensionless time $t$ with the dimensionless nonlinear time $\tau$ as indicated in eq (5b). In fact, this device for eliminating the diffusivity has been used before $[5,6]$. The same device would also eliminate $a$ from the equation if it were a function of the surface temperature $T(t)$ 
Eq- No.

$\left.(\mathbf{a}, \mathbf{b}) \quad \nabla * \cdot\left(k^{*} \nabla * \theta^{*}\right)=e^{*} c *\left[\partial \theta^{*} / \partial t^{* *}\right)^{*}+w^{*} \cdot \nabla * \theta^{*}\right]=e^{*} c *\left(\partial \theta^{*} / \partial t^{*}\right)_{r}$

(2)

$$
\nabla \cdot(k \nabla \theta)=\operatorname{ec}(\partial \theta / \partial t)
$$

$$
d \eta=c(\theta) d \theta
$$

$(4 \mathrm{a}, \mathrm{b}, \mathrm{c}) \quad \nabla \cdot(\mathrm{a} \nabla \eta)=\left(\mathrm{a}_{\eta} / \partial_{\mathrm{t}}\right)_{\mathrm{r}} ; a=k / \mathrm{ec} ; \mathrm{e}=\mathrm{constant}$

$(5 \mathrm{a}, \mathrm{b}) \quad \nabla^{2}{ }_{\eta}+\nabla \cdot u=\left(a_{\eta} / \mathrm{a}_{\tau}\right)_{r} ; d \tau=a(T[t]) d t=a(t) d t$

$(6 \mathbf{a}, \mathbf{b}) \quad u \equiv-\left[1-\frac{a(\theta)}{a(T)}\right] \nabla \eta ; \nabla-u \equiv q_{\mathrm{elf}}$

$$
\frac{\partial^{2}(r \eta)}{\partial r^{2}}-\frac{\partial(m)}{\partial \tau}=-r q_{\text {eff }}=\frac{1}{r} \frac{\partial}{\partial r}\left\{\left[\mathrm{I}-\frac{a(\theta)}{a(T)}\right] r^{2} \frac{\partial \eta}{\partial r}\right]=0
$$

$(8 \mathrm{a}, \mathrm{b}) \quad[\eta(r, \tau)]_{r=\mathrm{I}}=h(\tau) ;\left(\frac{\partial \eta}{\partial r}\right)_{r=1}=\frac{1}{3} \frac{d H}{d \tau} \equiv \frac{1}{3} H^{(1)}$

(8c)

$$
H \equiv \eta_{a x}=\int_{0}^{1} \rho \eta r^{2} d r / \int_{0}^{1} \rho r^{2} d r=3 \int_{0}^{1} \eta r^{2} d r
$$

rather than of the interior temperature $\Theta(r, t)$. Even in the latter ease, however, introduction of a nonlinear time $\tau$ based on $\alpha(T[t])$ succeeds in elininating most of the a-dependence from the equation, as shown by eqs (5a) and (6a). What remains in the equation is a very small effective heat source density term $q_{e f f}$ which has the form of the divergence of an effective heat flux $u$ defined in eq (6a). This heat flux vanishes at the surface of the sample, and for this reason, when Gauss' theorem is applied to eq (5a). in order to arrive at the expression given in eq $(8 \mathrm{~b})$ for the tirme-rate of total enthalpy change, the term involving $u$ makes no contribution. Therefore, because eq (8bi). leads to the equation (eq (19) of table V) from which the rest of the analysis follows, it is evident that, at least to first order, the introduction of the nonlinear time $\tau$ has succeeded in reducing the problem to the solution of the universal equation that results if $\mathrm{q}_{\text {eff }}=0 \mathrm{in}$ eq $(7)$. The analysis of this paper is based on this approximation. If more accuracy should be required, then the solution for $\eta(t, \tau)$ that is given in eq (9) or eq (12) of table III could be substituted into the right-hand side of eq (7), and an additive correction to $\eta$ could be found which in turn would lead to an additive correction to $\dot{H}$ which could be introduced into the calorimetric equations of section 4 . The way this wauld be done is explained in section 5 .

The solution to eq (7) (with 0 on the right-hand side) that satisfies the boundary condition stated in eq (8a) can be written in the form of $\mathrm{eq}(9)$ in table HII. The polynomials $p_{n}$ (r) are characterized by the property stated in eq (10a), and can be generated by successive integration. The first four polynomials are given in eq (I I), and are plotted in figure 1 . The fact that eq $(9)$ does indeed satisfy eq (7) (with 0 on the right-hand side) can be directly confirmed using the property stated in eq ( $\mathrm{I} 0 \mathrm{a}$ ).

As indicated in eq (12), the solution can also be expressed in terms of the odd-order Bernoulli polynomials $\mathbf{B}_{2 n+1}(x)$ where $x=1 / 2(1-r)$. The properties of these polynomials that are necessary to verify that eq (12) is indeed the desired solution of eq $(7)$ are stated in eqs (13) and (14). (See for example, pp. 804-811 of Ref. [14] or pp. 19, 25-29 of Ref. [15].).

Ar explicit expression for $H^{(D)} \equiv d H / d \tau$ in terms of $h^{(n)} \equiv d^{\mathrm{a}} h / d \tau^{\mathrm{m}}$ can be derived by substituting eq (T 2) into the left-hand' side of eq (8b) and using the relation stated in eq (16), of table IV between the even-order Bernoulli numbers $B_{2 \pi}$ and the Riermann Zeta funetion $\xi\left(2_{n}\right)$. The resulting relation is given in eq (19) af table $V$. $U$ sing the numerical values for $\xi(2 n)$, that are given in table $I V$, eq (20) results, which can be then inverted to yield eq (21), which will play an important role in section 4.

The expansion given in eq (19) assumes that $h(\tau)$ is an analytic function all of whose derivatives exist. If in addition it remains finite for all $\tau$, it can be shown that eq (19) is equivalent to the integral equation given in eqs. 
TABLE 111. Surface-Driven Solution for Interior Enthalpy Density.

Eq. No

$(9 \mathrm{a}, \mathrm{b}) \quad \eta(r, \tau)=h(\tau)+\sum_{n=1}^{\infty}(-1)^{n} p_{n}(r) h^{(n)}(\tau) ; h^{(n)} \equiv d^{n} h / d \tau^{n}$

$(10 \mathrm{a}, \mathrm{b}) \quad d^{2}\left(r p_{n}\right) / d r^{2}=-r p_{n-1}$ or $\nabla^{2} p_{n}=-p_{n-1}$

(11a) $\quad p_{1}=\left(1-r^{2}\right) / 6$

(11b) $\quad p_{2}=\left(7-10 r^{2}+3 r^{4}\right) / 360$

(11c) $\quad p_{3}=\left(31-49 r^{2}+21 r^{4}-3 r^{6}\right) / 15,120$

(11d) $\quad p_{4}=\left(381-620 r^{2}+294 r^{4}-60 r^{6}+5 r^{8}\right) / 1,814,400$

\section{Cf. Fig. 1}

$(12 \mathrm{a}, \mathrm{b}) \quad \eta(r, \tau)=h(\tau)-\frac{1}{r} \sum_{n=1}^{\infty} \frac{2^{2 n+1}}{(2 n+1) !} B_{2 n+1}(x) h^{(n)}(\tau) ; x \equiv \frac{1}{2}(1-r)$

$(13 \mathrm{a}, \mathrm{b})\left[B_{2 n+1}(x)\right]_{r=0}=B_{2 n+1}\left(\frac{1}{2}\right)=0:\left[B_{2 n+1}(x)\right]_{r=1}=B_{2 n+1}(0)=0$

$(14 a, b)$

$$
\frac{d B_{2 n+1}(x)}{d r}=-\frac{1}{2}(2 n+1) B_{2 n}(x) ; \quad \frac{d^{2} B_{2 n+1}(x)}{d r^{2}}=\frac{1}{4}(2 n+1)(2 n) B_{2 n-1}(x)
$$

(22a) and (23) of table VI. The kernel $\Gamma$ of the convolution integral defined in eq (23) is an effective memory function that weights the very recent past most heavily and totally forgets events that happened more than half a natural time unit in the past. This memory function is defined by eq (24) and is plotted in figure 2. Its argument is defined by eq (27a), and as shown in eqs $(27 \mathrm{~b}$ and c) can be expressed in terms of $\alpha(t)$ and the difference $\left(t-t^{\prime}\right)$ between the present time $t$ and some past time $t^{\prime}$. Figure 2 shows that for $\zeta$ less than 0.1 natural time units the simple function $\tilde{\Gamma}$ defined by eq $(25 \mathrm{~b})$ is essentially indistinguishable from $\Gamma$. For larger $\zeta$, the first term in the summation of eq (24) should serve to represent $\Gamma$ with sufficient accuracy for most purposes. As eq (26) indicates, the normalization of $\Gamma$ is such that if $h^{(2)}$ is constant, then the retrospective weighted average $h^{(2)}$ defined by eq (23) will just be equal to $h^{(2)}$. If, however, $h^{(2)}$ varies drastically during half a natural time unit, which could be the case when a sample first starts to cool, then the weighted average $\overline{h^{(2)}(t)}$ will differ markedly from the instantaneous value $h^{(2)}(t)$. In such a case the integral eq (22a) will be more aecurate than the equivalent truncated expansion given in eq (20). The series expansion (19) can be derived from the integral equation defined by eqs $(22 a)$ and $(23 b)$ by expressing $h^{(2)}(\tau-\zeta)$ as a Taylor expansion about $\tau$, integrating by parts, and making use of the definition of $\zeta(2 n)$ given in eq (15) in table IV.

Finally, it should be noted that it is evident from eq (23b) that when $\overline{h^{(2)}(\tau)}$ is differentiated with respect to $\tau$, the differentiation can be taken inside the integration, from which it follows that eq (22b) results from differentiation of eq (22a). Obviously, an infinity of such equations can be generated by repeated differentiation.

TAвLE IV. Riemann Zeta Function for Even-Integer Argument.

Eq. No.

$\begin{array}{ll}\text { (15) } & \zeta(2 n) \equiv \sum_{m=1}^{\infty} m^{-2 n} \\ \text { (16) } & B_{2 n} \equiv\left[B_{2 n}(x)\right]_{x=0}=\frac{(-1)^{n-1}(2 n) !}{2^{2 n-1}} \frac{\zeta(2 n)}{\pi^{2 n}}\end{array}$

\begin{tabular}{|lll|}
\hline & & \\
\hline $2 n$ & $\zeta(2 n) / \pi^{2 n}$ & $\zeta(2 n)$ \\
\hline 2 & $1 / 6$ & 1.64493 \\
4 & $1 / 90$ & 1.08232 \\
6 & $1 / 945$ & 1.01734 \\
8 & $1 / 9450$ & 1.00407 \\
10 & $1 / 93,555$ & 1.00099 \\
\hline
\end{tabular}


Eq. No.

$(17 \mathrm{a}, \mathrm{b}, \mathrm{c}) \quad H^{(1)} \equiv d H / d \tau ; \dot{H} \equiv d H / d t ; H^{(1)}=\dot{H} / a(t)$

$(18 \mathrm{a}, \mathrm{b}) \quad h^{(1)}=c(\tau) T^{(1)}(\tau)=c(t) \dot{T}(t) / \alpha(t) ; h^{(n)} \equiv d^{n} h / d \tau^{n}$

(19)

$$
\begin{aligned}
& H^{(1)}(\tau)=-6 \sum_{n=1}^{\infty}(-1)^{n}\left[\zeta(2 n) / \pi^{2 n}\right] h^{(n)}(\tau) \\
& H^{(1)}(\tau)=h^{(1)}-\frac{1}{15} h^{(2)}+\frac{2}{315} h^{(3)}-\frac{1}{1575} h^{(4)}+\ldots \\
& h^{(1)}(\tau)=H^{(1)}+\frac{1}{15} H^{(2)}-\frac{1}{525} H^{(3)}+\frac{2}{23,625} H^{(4)}+\ldots
\end{aligned}
$$

Table VI. Total Rate of Energy Lass: Integral Representation.

Eq. No.

(22a,b) $H^{(1)}=h^{(1)}-\frac{1}{15} \overline{h^{(2)}} ; H^{(2)}=h^{(2)}-\frac{1}{15} \overline{h^{(3)}} ;$ etc.

$(23 \mathrm{a}, \mathrm{b}) \quad \overline{h^{(2)}} \equiv \int_{-\infty}^{\tau} \Gamma\left(\tau-\tau^{\prime}\right) h^{(2)}\left(\tau^{\prime}\right) d \tau^{\prime}=\int_{0}^{\infty} \Gamma(\zeta) h^{(2)}(\tau-\zeta) d \zeta$

$$
\Gamma(\xi) \equiv 90 \sum_{m=1}^{\infty}(m \pi)^{-2} \exp \left[-(m \pi)^{2} \zeta\right]
$$

(25a) $\Gamma(\zeta) \doteq \tilde{\Gamma}(\zeta)$ for $0 \leqslant \zeta \leqslant 0.1$

\section{(Cf. Fig. 2)}

(25b) $\tilde{\Gamma}(\xi) \equiv 15[1-3(2 \sqrt{\zeta / \pi}-\xi)]$

$(26 \mathrm{a}, \mathrm{b}) \quad \int_{-\infty}^{\tau} \Gamma\left(\tau-\tau^{\prime}\right) d \tau^{\prime}=\int_{0}^{\infty} \Gamma(\zeta) d \zeta=1$

(27a) $\quad \zeta \equiv \tau-\tau^{\prime}=\int_{t^{\prime}}^{t} a\left(t^{\prime \prime}\right) d t^{\prime \prime}$

(27b) $\quad \zeta\left(\alpha ; t-t^{\prime}\right)=\sum_{n=0}^{\infty} \frac{(-1)^{n}}{(n+1) !}\left(d^{n} a / d t^{n}\right)\left(t-t^{\prime}\right)^{n}$

(27c) $\quad \zeta\left(\alpha ; t-t^{\prime}\right)=\alpha(t)\left(t-t^{\prime}\right)-\frac{1}{2} \dot{\alpha}(t)\left(t-t^{\prime}\right)^{2}+\frac{1}{6} \ddot{\alpha}(t)\left(t-t^{\prime}\right)^{3}-\ldots$

\section{The Predictive Problem}

For the purpose of estimating radiative cooling times and the relative magnitudes of the terms in eq $(21)$, from which the calorimetric equations of section 4 are derived, it is useful to solve the post-transient predictive problem for the case of constant parameters. In such a case the simplifications indicated in eqs (28a-d) of table VII occur. All of the equations of tables $\mathrm{V}$ and VI are still valid. ln particular, $\dot{H}=H^{(1)}$ must satisfy eq (19), but in addi- tion it must satisfy the Stefan-Boltzmann radiation law which means that the left-hand side of eq (19) must be replaced by $T^{4}$ multiplied by a proportionality constant involving the Stefan-Boltzmann constant $\sigma^{*}=\hat{\sigma}$. It is easy to show that if the unit temperature $\hat{T}$ is defined as shown in eq $(29 \mathrm{~b})$, the proportionality constant on the left side of the specialized form of eq (19) will by unity, with the result that the equation has the form given in eq (31). A significant feature of this equation is that it is universal in the sense that it applies to spherical samples of all sizes made of any material whose thermal 


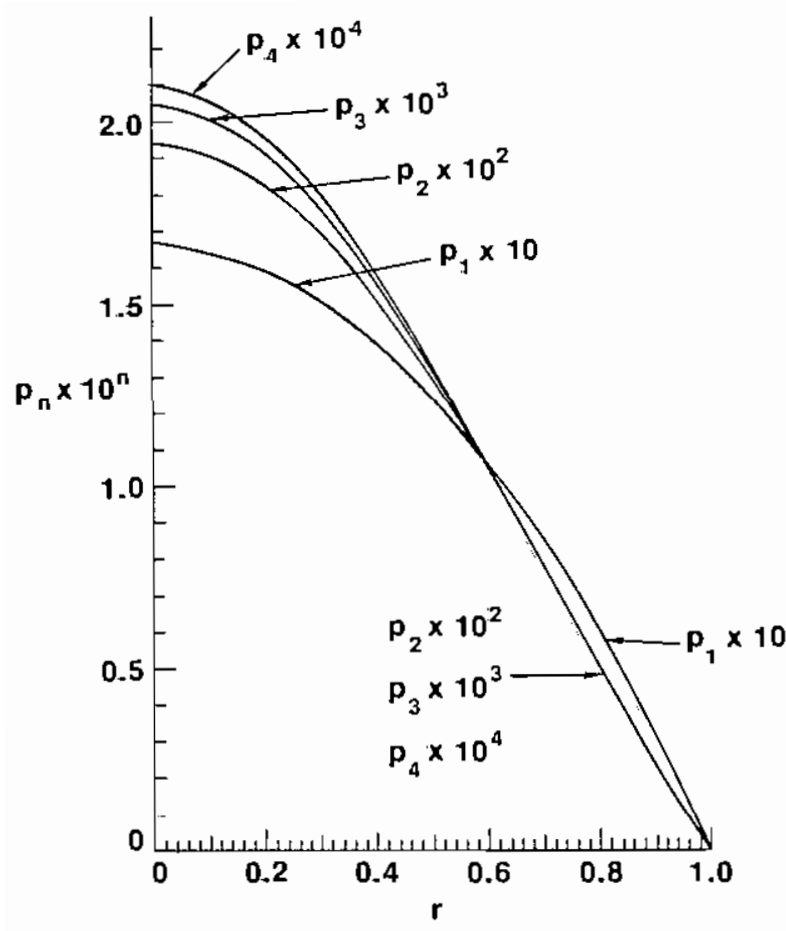

Figure 1. Dimensionless polynomials $p_{n}(r)$ defined in eqs $\{1$ la-d).

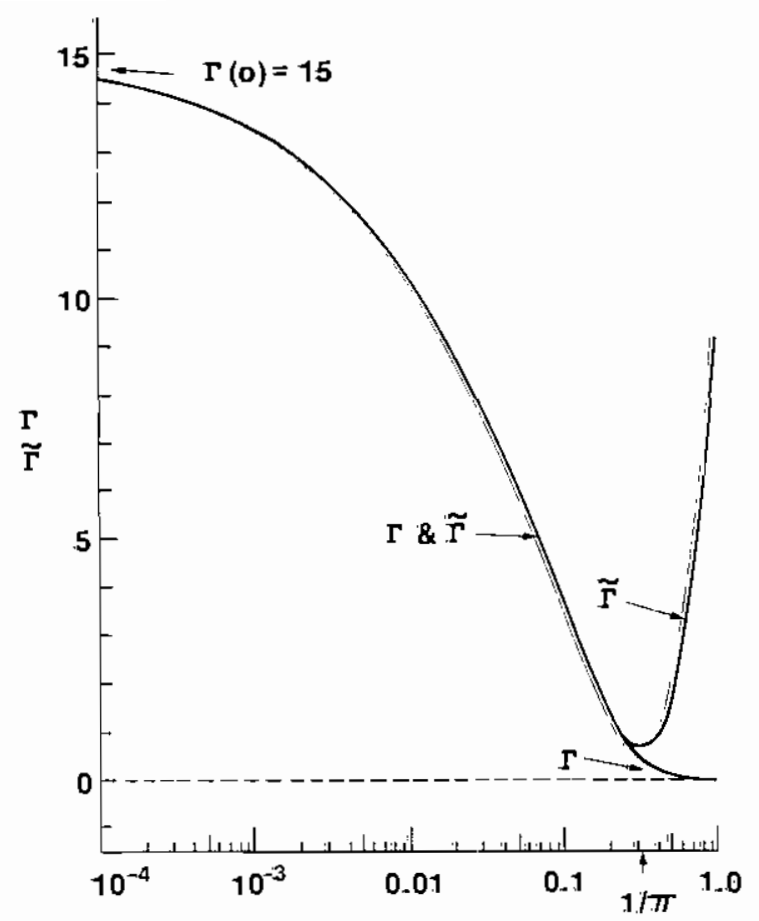

FIGURE 2. Dimensionless kernel $\Gamma(\zeta)$ in convolution integral delined in eq (23), and approximate kernal $\widetilde{\Gamma}(\xi)$ defined in eq (25b), both as functions of the dimensionless argument $\xi \equiv \pi-\tau^{\prime}$.

Table VIL. Equations for Predictive Problem.

Eq. No.

(28a,b,c,d) $\quad c=\alpha=k=R=\varepsilon=\varrho=1 ; \tau=t ; \eta=\theta ; h=T$

$(29 \mathrm{a}, \mathrm{b}) \quad T=T^{*} / \hat{T} ; \hat{T} \equiv(\hat{k} / \hat{R} \sigma \hat{\varepsilon})^{1 / 3}=\left(k^{*} / \mathrm{R}^{*} \sigma^{*} \varepsilon^{*}\right)^{1 / 3}=\left(\mathrm{Q}^{*} \alpha^{*} c^{*} / R^{*} \sigma^{*} \varepsilon^{*}\right)^{1 / 3}$

$(30 \mathrm{a}, \mathrm{b}) \quad \ddot{H} \equiv d H / d t=-3 T^{4} ;(\partial \eta / \partial r)_{r=1}=(\partial \theta / \partial r)_{r=1}=-T^{4}$

$T^{4}=2 \sum_{n=1}^{\infty}(-1)^{\mathrm{n}}\left[\zeta(2 n) / \pi^{2 n}\right] d^{n} T / d t^{n}$

$T^{4}=-\frac{1}{3} \frac{d T}{d t}+\frac{1}{45} \frac{d^{2} T}{d t^{2}}-\frac{2}{945} \frac{d^{3} T}{d t^{3}}+\frac{1}{4725} \frac{d^{4} T}{d t^{4}}-\ldots$

$(33 \mathrm{a}, \mathrm{b}) \quad d T / d t=-3 T^{4}\left[1-\frac{4}{5} T^{3}+\frac{4}{25} T^{6}+\frac{144}{85} T^{9}+\ldots\right] ; d H / d t=-3 T^{4}$

(34a,b) $\quad d^{2} T / d t^{2}=36 T^{7}\left[1-\frac{11}{5} T^{3}+\frac{42}{25} T^{6}+\ldots\right] \quad ; d^{2} H / d t^{2}=36 T^{7}\left[1-\frac{4}{5} T^{3}+\frac{4}{25} T^{6}+\ldots\right]$

$(35 \mathrm{a}, \mathrm{b}) \quad d^{3} T / d t^{3}=-756 T^{10}\left[1-\frac{138}{35} T^{3}+\ldots\right] \quad ; d^{3} H / d t^{3}=-756 T^{1.0}\left[1-\frac{68}{35} T^{3}+\ldots\right]$

$(36 \mathrm{a}, \mathrm{b}) \quad d^{4} T / d t^{4}=22,680 T^{13}-\ldots \quad ; d^{4} H / d t^{4}=22,680 T^{13} \ldots \ldots$

$t(T) \doteq \frac{1}{9}\left(T^{-3}-1\right)-\frac{4}{15} \ln T+\frac{4}{75}\left(1-T^{3}\right)$

$T(t) \doteq\left[(1+9 t)-\frac{4}{15} \ln (1+9 t)-\frac{108}{25} \frac{t}{(1+9)}\right]^{-1 / 3}$

(Cf. Fig. 3)

$T(t)=\left[-\frac{1}{3} \dot{T}(t)+\frac{1}{45} \int_{0}^{\infty} \Gamma(\xi) \ddot{T}(t-\zeta) \mathrm{d} \zeta\right]^{1 / 4}$ 
parameters are constants. It is possible to solve this equation by truncating it at the fourth derivative as shown in eq (32), and solving the truneated equation for $d T / d t$ in terms of $T$ by starting with the approximation $d T / d t \doteq-3 T^{4}$ and iterating until a self-consistent set of expressions for the first four derivatives results. These expressions are given in eqs ( $33 \mathrm{a}-36 \mathrm{a})$. By differentiating eq (30a) and making use of eqs (33a-36a), the expressions for $d^{\mathrm{n}} H / d t^{\mathrm{n}}$ given in eqs $(34 \mathrm{~b}-36 \mathrm{~b})$ can be derived. The 8 expressions in eqs $(33-36)$ will be used in section 4 to estimate the range of vahdity of the calorimetric formulas derived there. Numerical estimates indicate that these expressions are aceurate to within $1 \%$ so long as $T$ $\leqslant 1 / 2$. For larger $T$, it would be necessary to include higher order terms in the expansion given in eq (3.1), and the numerical estimates indieate that for $T>3 / 4$ the convergence is so slow that this expansion has no practieal utility. Correspondingly, the calorimetrie equations derived in seetion 4 that are based on eq (21), which is derived from eq (19), cannot be expected to be accurate, even in a post-transient experiment, if the dimensionless surface temperature $T$ based on the unit temperature defined in eq $(29 b)$ is larger than $1 / 2$. If the sphere size and thermal parameters are sucb that $T>1 / 2$, then it will be necessary to use equations based on the integral equation defined by eqs (22a) and (23). In order to give a feeling for what sphere sizes and which materials will satisfy the condition $T<1 / 2$, the dimensionless temperatures $T_{\text {melting }}$ corresponding to the respective melting points of tungsten $(3650 \mathrm{~K})$ and uranium dioxide $(3150 \mathrm{~K})$ are given in table VIII for sphere radii that approximate the upper and lower limits that would most probably be considered for radiative-cooling calorimetry experiments. It is evident from this chart that for most praetical posttransient experiments, it should be possible to use ealorimetrie relations derived from the truncated expansion given in eq (21). Only in the ease of a large sample $(\hat{\mathbf{R}} \approx 1 \mathrm{~cm}$ ) of a poor thermal conduetor (such as uranium dioxide) might it be necessary to use an integral relation in order to analyze the results of a post-transient experi-

TaBLE VIII. Representative Valué for Unit Time and Unit Temperature.

\begin{tabular}{|c|c|c|c|c|}
\hline \multirow{2}{*}{$\hat{\mathbf{h}}$} & \multicolumn{2}{|c|}{ Tungaten } & \multicolumn{2}{|c|}{ Uranium Dioxide } \\
\hline & $0.1 \mathrm{~cm}$ & $1 \mathrm{~cm}$ & $0.1 \mathrm{~cm}$ & $1 \mathrm{~cm}$ \\
\hline $\begin{array}{l}\hat{\hat{r}} \\
\hat{T} \\
T^{*} \text { melting } \\
T_{\text {melting }}\end{array}$ & $\begin{array}{l}0.03 \text { ec } \\
37,000 \mathrm{~K} \\
3650 \mathrm{~K} \\
0.1\end{array}$ & $\begin{array}{l}3 \text { sec } \\
17,000 \mathrm{~K} \\
3650 \mathrm{~K} \\
0.2\end{array}$ & $\begin{array}{l}2 \text { aec } \\
8100 \mathrm{~K} \\
3150 \mathrm{~K} \\
0.4\end{array}$ & $\begin{array}{l}200 \mathrm{sec} \\
3800 \mathrm{~K} \\
3150 \mathrm{~K} \\
0.8\end{array}$ \\
\hline
\end{tabular}

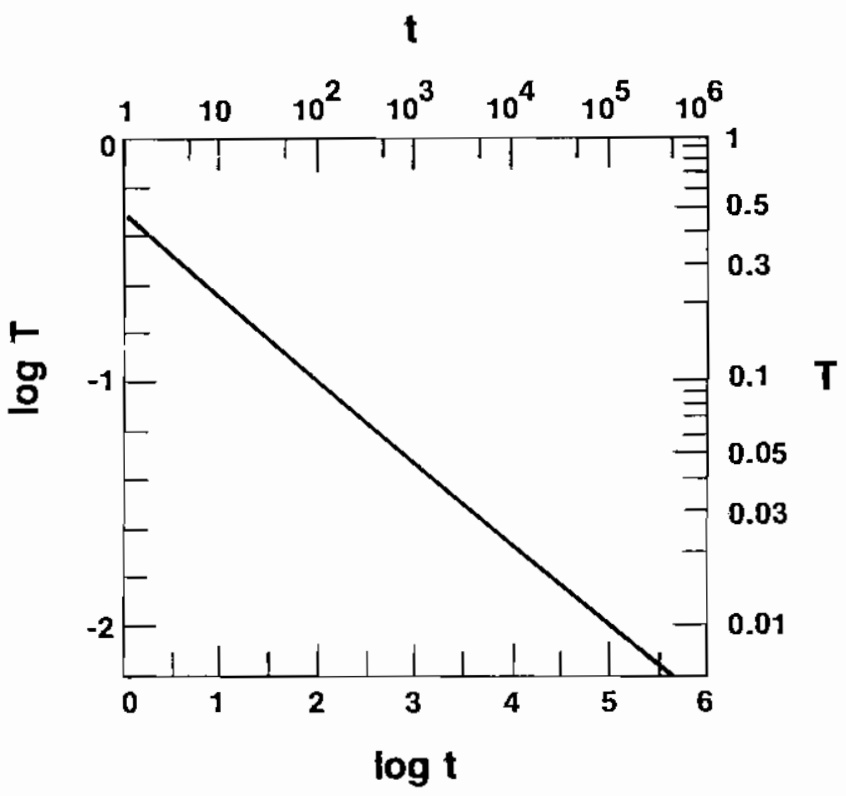

Figure 3. Universal temperature-time curve for post-transient radiative cooling.

ment. It ought to be noted, however, that this might be neeessary even in the case of a smaller sample of a better conductor if the observations are based on a transienttype experiment.

A universal post-transient cooling curve ean be derived by integrating eq (33a). The result is the expression for $t(T)$ given in eq (37). This ean be inverted to yield the expression for $T(t)$ given in eq (38). The cooling curve corresponding to these expressions is plotted in figure 3 . It is evident from this curve that the slope of $\ln T$ versus ln $t$ is almost, but not quite, constant. In fact, this slight variation in slope is related to the thermal condnetivity of the sample. It can be shown that for initial and final temperatures $T_{i}^{*}$ and $T_{f}^{*}$

$$
\begin{gathered}
k^{*}=\frac{4}{15} \hat{R}_{\hat{\sigma} \hat{\varepsilon}}\left(\frac{T_{i}^{* 3}-T_{f}^{* 3}}{s_{i}-s_{f}}\right) \\
s_{i}=\left(\frac{d \operatorname{In} T^{*}}{d \operatorname{In} t^{*}}\right)_{T_{i}^{*}} ; s_{f}=\left(\frac{d \operatorname{In} T^{*}}{d \operatorname{In} t^{*}}\right)_{T_{f}^{*}}
\end{gathered}
$$

This equation cannot be used for determining thermal condnctivity from observation of post-transient radiative cooling, because it assumes that the specific heat is constant throughout the cooking, whereas in all probability the $\ln T^{*}$ versus $\ln t^{*}$ curve for a real sample would have much more curvature than the one shown in figure 3 , and most of this curvature would be caused by the temperature dependence of the speeific heat. The real significance of eq (40) is that it (together with Fig. 3) illnstrates how difficult it is to make a reliable determination of thermal eonductivity (or diffnsivity) from observations of post-transient radiative cooling, 
especially when these observations are made for temperatures near the lower end of the curve shown in figure 3 . In contrast, it is very easy to determine the specific heat in this range since to a good approximation it will be given by $c \doteq \dot{H} / \dot{T}$. For an accurate determination of thermal conductivity it will probably be necessary to use a sphere that is large enough so that the dimensionless temperatures involved fall well above those shown in figure 3 . In such a case it would be necessary to analyze the data using the integral expressions given in section 4 , rather than the truncated expansions.

It should be noted that when the expressions given in eqs $(33 a-36 a)$ are substituted into eq (9a) taking the simplifications stated in eqs $(28 \mathrm{a}-\mathrm{d})$ into account, a complete solution for the interior surface-driven solution in terms of the surface temperature $T$ results. If the expression for $T(t)$ given in eq $(38)$ is substituted into this, an explicit expression for the interior temperature field $\Theta(r, t)$ results. If it were desired to extend the validity of this solution to values of $T$ larger than $1 / 2$, this could be done by using the integral equation given in eq (39) in table VII as the basis for an iterative solution. Equation (39) was derived from the integral equation defined by eqs (22a) and (23), making use of eq (30a). The idea of reducing the problem of solving for the interior temperature field to the problem of solving an integral equation involving only the time dependence of the surface temperature is not new. It has been done for a semiinfinite medium with a plane surface [16]. The integral equation that resulted was derived from the diffusion convolution integral mentioned in section 1 . However, this approach is not appropriate for the present problem because, as noted in section 1 , the diffusion convolution integral represents the temperature field in an infinite medium surrounding a spherical cavity, rather than the field within a finite spherical medium.

\section{The Calorimetric Problem}

In adapting the expressions derived in section 2 to the problem of deducing the specific heat and the thermal diffusivity from observational data, the choice made for the unit temperature $\hat{T}$ can be arbitrary. It is not necessary to use the unit temperature defined by eq (29b) of table VII, although this choice is appropriate for the purposes of designing an experiment, and for determining whether the various expressions derived. in section 4.1 from eq (21) are accurate, or whether it is necessary to use the alternative integral relations discussed in section 4.2 .

Once it is a question of analyzing existing data, however, it would generally be more convenient to define
$\hat{T}$ so that the dimensionless temperature $T$ is close to unity. If the thermal parameters are already known for the cold end of a post-transient cooling run, then it would be natural to choose this cold temperature as the unit temperature, and correspondingly the unit specific heat and unit diffusivity would be chosen to be equal to the known valucs of these parameters at this cold temperature. If the true values are not known, then estimates would suffice. Nowhere in the analysis is it assumed that these estimates are close to the true values. For example, if one were analyzing data for samples of different materials, it might be most convenient to make a single choice of unit quantities to be used for all of the different materials.

In all of the expressions given below, $\dot{H}(t) \equiv d H / d t$ and $T(t)$ are regarded as given functions of time that result from independent simultaneous observations made by two different instruments. If a reliable cooling law exists and is known, then $\dot{H}$ can be expressed as a function of $T$ and eliminated from the equations. In the case of radiative cooling, this would require that the temperature dependence of the total hemispherical emissivity $\varepsilon(T)$ be known.

\subsection{Truncated Expansions}

The calorimetric formulas, which were the principal objective of this analysis, are given in eqs (41) and (42) of table IX. The expression for $c$ follows directly from eq (40), which is simply eq (21) of table V multiplied by $\alpha(t)$. The expression for $\alpha$ was derived from the ratio of the time derivative of eq (40a) to eq (40a) itself. Both expressions for $c$ and $\alpha$ have the form of a power series in an expansion parameter $\xi=1 / 15 \alpha$. The coefficients of these power series are functions of the four quantities defined in eqs $(43 \mathrm{a}-\mathrm{d})$, the leading terms of which are ratios of different time derivatives of $T$ and $H$. Equations (44) and (45) give approximate expressions for $c$ and $\alpha$ that are valid in the limiting case in which only the leading terms in the expansions must be retained, and $c$ and $\alpha$ are essentially constant.

In the discussion that follows, it will be assumed that the unit of diffusivity $\hat{\alpha}$ has been chosen so that for the data under consideration the dimensionless diffusivity $\alpha$ is of order unity. Then $\xi \approx 1 / 15$. (If a different choice of $\hat{\alpha}$ were made, the change in $\xi$ would be compensated by changes in the values of the quantities defined in eqs (43a-d.) Because the expansion parameter $\xi$ involves $\alpha$, and the coefficients in the expansions for $c$ and $\alpha$ involve $\dot{c}$ and $\dot{\alpha}$, it is evident that eqs (41) and (42) must be solved iteratively, with the first iteration based on the assumption that $\xi=\dot{c}=\dot{\alpha}=0$. The range of convergence of this procedure can be estimated by using the 
expressions for $d^{\mathrm{n}} T / d t^{\mathrm{n}}$ and $d^{\mathrm{n}} H / d t^{\mathrm{n}}$ given in eqs (3336 ) of table VII to evaluate all of the terms in eqs (41) and (42). When this is done the following expressions result:

$$
\begin{aligned}
& A=-12 T^{3}\left(1-\frac{7}{5} T^{3}+\frac{2}{5} T^{6}+\ldots\right) \\
& B=-12 T^{3}\left(1-\frac{4}{5} T^{3}+\frac{4}{25} T^{6}+\ldots\right) \\
& C=252 T^{6}\left(1-\frac{68}{35} T^{3}+\ldots\right) \\
& D=-7560 T^{9}+\ldots \\
& \frac{D-A C}{A-B}=-630 T^{3}+\ldots \\
& \frac{A D}{A-B}=12,600 T^{6}+\ldots \\
& c_{o}=\dot{H} / \dot{T}=1+\frac{4}{5} T^{3}+\frac{12}{25} T^{6} \\
& \quad+\frac{16}{175} T^{9}+\ldots \\
& \alpha_{0}=\frac{1}{15}\left(\frac{C-A B}{A-B}\right)=1-\frac{6}{5} T^{3}+\ldots
\end{aligned}
$$

When these expressions and $\xi \approx 1 / 15$ are substituted into the right-hand side of eq $(41 \mathrm{a})$, it reduces to $1+$ $0\left(T^{12}\right)$ which (since the left-hand side is $c=1$ ) is just the identity that is to be expected in view of the fact that eq (4la) is simply a reformulation of the same equation from which eqs (33-36) were derived. Similarly, the right-hand side of eq (42) reduces to $1+0\left(T^{6}\right)$. Thus (to the accuracy of the truncation) the leading factors in eqs (41 a) and (42), which are now expressed by eqs (48) and (49), are just the reciprocals of the respective square brackets on the right-hand sides of eqs (4la) and (42). For this reason, the speed of convergence of the calorimetric formulas can be estimated by inspecting eqs (48) and (49). These indicate acceptably rapid convergence for $T<1 / 2$ which, of course, is the same range of convergence that was noted in section 3 for the validity of the iterative solution of the predictive problem.

In the case of the approximate limiting expressions given in eqs (44) and (45), all of the terms of eqs (41 a) and (42) that involve $\xi$ were thrown away, and only the leading terms were retained. In addition, all of the terms of eqs ( $43 \mathrm{a}-\mathrm{d})$ involving $\dot{c}$ and $\dot{\alpha}$ were thrown away. The validity of this latter approximation can be answered only on a case-by-case basis, but the validity of ignoring the terms involving $\xi$ can be estimated by means of eqs (48) and (49) since the terms involving $T$ represent the error in these formulas, because in this case the correct values of $c$ and $\alpha$ are unity. In the chart below the magnitudes of the two leading error terms are listed for several values of $T$.

\begin{tabular}{lll}
\hline \hline $\mathrm{T}$ & $\frac{4}{5} T^{3}$ & $\frac{6}{5} T^{3}$ \\
\hline $1 / 2$ & 0.10 & 0.15 \\
$1 / 4$ & 0.013 & 0.019 \\
0.1 & 0.0008 & 0.001 \\
\hline
\end{tabular}

This chart shows that for $T<1 / 4$, the error is less than 2 percent, and becomes increasingly smaller the smaller $T$ becomes, i.e, as $T$ enters the extreme post-transient regime. Even for $T=1 / 2$, the error is not so large as to destroy the usefulness of eqs (44) and (45) for generating the first iterative solution for $c(t)$ and $\alpha(t)$ which is then substituted into the right-hand sides of the more accurate formulas given in eqs (41) and (42). If $\alpha_{0}$ is constant, it follows from eq (45d) that $\dot{H} \propto \exp \left[-15 \alpha_{0} \mathrm{t}\right]$, so that in the extreme post-transient regime $\alpha_{0}$ can be estimated by fitting the observed function $\dot{H}(t)$ to an exponential decay.

It should be noted that, in order to make these estimates, it has been necessary to define the dimensionless $T$ in the manner indicated in eq (29) of table VII. This automatically takes the sphere size and thermal parameters of the sample into account. However, for an actual application of the calorimetric formulas of table IX, it is not necessary to do this. One may use any convenient scaling factor to define the dimensionless $T$. The validity of the formulas would then be indicated directly by the convergence behavior of the numerical iteration process.

The solutions of eqs $(41)$ and $(42)$ are $c(t)$ and $\alpha(t)$. However, because $T(t)$ is known from observation, these solutions can be converted into $c(T)$ and $\alpha(T)$, which are the desired expressions for the temperatyre dependence of the thermal parameters.

\subsection{Integral Relations}

If a numerical application of the calorimetric formulas shows that the $\xi^{3}$ term in eq $(42)$ is comparable in magnitude with the $\xi^{2}$ term, or if the $\xi^{2}$ term in eq (41a) is comparable with the $\xi$ term, this is an indication that the neglected higher-order terms are not really negligible, and that the calculated functions $\alpha(t)$ and $c(t)$ are not reliable. One could, of course, include higher-order terms in the equations, but truncation must occur at some point, so the net result would be only a slight extension of the range of validity of the equations. Moreover, the higher-order terms involve higher derivatives of $T(t)$ 
Eq. No.

$$
\begin{gathered}
(40 \mathrm{a}, \mathrm{b}) \quad \dot{h}=c \dot{T}=\dot{H}+\frac{1}{15}\left[\frac{d H^{(1)}}{d t}-\frac{1}{35} \frac{d H^{(2)}}{d t}+\frac{2}{1575} \frac{d H^{(3)}}{d t}+\ldots\right] ; \\
{\left[H^{(1)}=\frac{\dot{H}}{\alpha}, H^{(2)}=\frac{1}{\alpha} \frac{d H^{(1)}}{d t}, H^{(3)}=\frac{1}{\alpha} \frac{d H^{(2)}}{d t}\right]}
\end{gathered}
$$

$(41 \mathrm{a}, \mathrm{b}) \quad c=(\dot{H} / \dot{T})\left[1+B \xi-\left(\frac{3}{7} C\right) \xi^{2}+\left(\frac{2}{7} D\right) \xi^{3}+\ldots\right] ; \xi \equiv 1 / 15 \alpha$

$$
\alpha=\frac{1}{15}\left(\frac{C-A B}{A-B}\right)\left[1-\frac{3}{7}\left(\frac{D-A C}{A-B}\right) \xi^{2}-\frac{2}{7}\left(\frac{A D}{A-B}\right) \xi^{3}+\ldots\right]
$$

(43a) $\quad A \equiv d \ln h(1) / d t=d \ln (c \dot{T} / \alpha) / d t=(\ddot{T} / \dot{T})+[(\dot{c} / c)-(\dot{a} / \alpha)]$

(43b) $\quad B \equiv d \ln H^{(1)} / d t=d \ln (\dot{H} / \alpha) / d t=(\dot{H} / \dot{H})-(\dot{\alpha} / \alpha)$

(43c) $\quad C \equiv \alpha^{2} H^{(3)} / H^{(1)}=(\ddot{H} / \dot{H})-3(\dot{\alpha} / \alpha)+\left[(\ddot{\alpha} / \alpha)+(\dot{\alpha} / \alpha)^{2}\right]$

(43d) $\quad D \equiv \alpha^{3} H^{(4)} / H^{(1)}=(\dddot{H} / \dot{H})-6(\dot{\alpha} / \alpha)(\ddot{H} / \dot{H})+\left[15(\dot{\alpha} / \alpha)^{2}-4(\ddot{\alpha} / \alpha)\right](\dot{H} / \dot{H})-\left[(\ddot{\alpha} / \alpha)+15(\dot{\alpha} / \alpha)^{3}\right.$

$\left.-10\left(\dot{\alpha} \bar{\alpha} / \alpha^{2}\right)\right]$

(44)

$$
c_{\mathrm{o}} \equiv(c)_{\xi=\dot{\mathrm{c}}=\dot{\alpha}=\mathrm{o}}=\dot{H} / \dot{T}
$$

$(45 \mathrm{a}, \mathrm{b}) \quad \alpha_{\mathrm{o}} \equiv(\alpha)_{\xi=\dot{c}=\dot{\alpha}=0}=\frac{1}{15}\left(\frac{C-A B}{A-B}\right)_{\dot{e}=\dot{\alpha}=0}=\frac{1}{15} \frac{(\dot{T} \ddot{H}-\ddot{T} \ddot{H})}{(\ddot{T} \dot{H}-\dot{T} \ddot{H})}$

$(45 \mathrm{c}, \mathrm{d})$

$$
=-\frac{1}{15}\left|\begin{array}{ll}
\vec{T} & \ddot{H} \\
\ddot{T} & \vec{H}
\end{array}\right|\left|\begin{array}{ll}
\dot{T} & \dot{H} \\
\ddot{T} & \ddot{H}
\end{array}\right|^{-1}=-\frac{1}{15} \frac{d}{d t}\left(\frac{\ddot{H}}{\dot{T}}\right) / \frac{d}{d t}\left(\frac{\dot{H}}{\dot{T}}\right)
$$

Table X. Calorimetric Problem: Integral Relations.

Eq. No.

(50)

$$
\alpha(t)=\{[(\ddot{h}-\ddot{H}) /(\vec{h}-\dot{H})]-(\dot{\alpha} / \alpha)\} \overline{\left[h^{(2)} / \overline{h^{(3)}}\right.}
$$$$
\vec{h}(t)=\dot{H}+\frac{1}{15}\{[(\ddot{h}-\ddot{H}) /(\dot{h}-\dot{H})]-(\dot{\alpha} / \alpha)\}\left[\left(\overrightarrow{h^{(2)}}\right)^{2} / \overrightarrow{h^{(3)}}\right]
$$

$$
\overline{h^{(2)}} \equiv \int_{-\infty}^{T} \Gamma \frac{d}{d t^{\prime}}\left[\frac{\dot{h}\left(t^{\prime}\right)}{\alpha\left(t^{\prime}\right)}\right] d t^{\prime}
$$

$$
\overline{h^{(3)}} \equiv \int_{-\infty}^{t} \Gamma \frac{d}{d t^{\prime}}\left\{\frac{1}{\alpha\left(t^{\prime}\right)} \frac{d}{d t^{\prime}}\left[\frac{\dot{h}\left(t^{\prime}\right)}{a\left(t^{\prime}\right)}\right]\right\} d t^{\prime}
$$

$$
\begin{aligned}
& \Gamma \equiv 90 \sum_{m=1}^{\infty}(m \pi)^{-2} \exp \left[-(m \pi)^{2} \zeta\right] \\
& \zeta \equiv \int_{t^{\prime}}^{t} \alpha\left(t^{\prime \prime}\right) d t^{\prime \prime}=\alpha(t)\left(t-t^{\prime}\right)-\frac{1}{2} \cdot(t)\left(t-t^{\prime}\right)^{2}+\frac{1}{6} \ddot{\alpha}(t)\left(t-t^{\prime}\right)^{3}-\ldots
\end{aligned}
$$

$(57 \mathrm{a}, \mathrm{b}) \quad c(\mathrm{~T})=c(t[T]) ; \alpha(T)=\alpha(t[T])$ 
and $\dot{H}(t)$, and the error involved in extracting these from the experimental data becomes ever greater the higher the order of differentiation. For these reasons, it is better to use an iterative procedure based on eqs (50) and (51) of table $X$ wbich involve the integrals defined in eqs (52) and (53). These equations were derived from eqs (22a) and $(22 \mathrm{~b})$ of table VI. Because $\ddot{h}(t)=c(t) \vec{T}(t)$, eq $(51)$ is really an equation for $c(t)$, but it is simpler to regard $h(t)$ as the unknown function and, after this has been found, to invoke eq $(5.6)$ to find $c(t)$, and eqs $(57 \mathrm{a}, \mathrm{b})$ to find $c(T)$ and $\alpha(T)$. The kernel $\Gamma$ of the integrals is defined by eqs (.54) and (5.5), but the approximation based on eq (25b) and discussed at the end of section 2 would simplify the calculations. Inasmuch as the temperature dependence (and hence the time dependence) of $\alpha$ is usually weak, and (as Fig. 2 indicates) $\Gamma$ will usually vanish in a time interval that is short compared with the time required for $\alpha$ to change by a significant amount, in all but the most extreme of transient experiments it would be justified to drop all but the first term in the expansion for $\zeta$ given in eq (.55).

The iteration could be started with an $\alpha(t)$ calculated from eq (45) and an $\dot{h}(t)=c(t) \dot{T}(t)$ where $c(t)$ is found from eq (44). These approximate functions would be substituted into the right-hand sides of eqs $(50)$ and (51), which would yield new (presumably improved) approximations. Qnestions of convergence and numerical stability of this procedure have not yet been investigated.

\section{Discussion}

The foregoing analysis took the temperature dependence of the specific heat fully into account, but the interior spatial variation of the diffusivity was neglected. This amounted to neglecting an effective heat source density in the Fourier equation, but it was pointed out in section 2 that this neglected term could be taken into account in an iterative fashion. This would give rise to an additive correction $\delta_{\alpha} \eta$ to the interior enthalpy density field. It was noted in section 2 that when Gauss' theorem is applied to the Fourier equation, the effective heat term makes no direct contribution to the resulting equation (eq $(8 \mathrm{~b})$ in table $\mathrm{II}$ ). It does make an indirect contribution, however, in the sense that it produces an additive correction to the radial derivative of the interior enthalpy field. Thus, eq $(8 b)$ must be replaced by

$$
\left[\partial\left(\eta+\delta_{\alpha} \eta\right) / \partial r\right]_{r=1}=\frac{1}{3} d\left(H+\delta_{\alpha} H\right) / d \tau
$$

where $H$ is given by eq $(8 \mathrm{c})$ and $\delta_{\alpha} H$ is given by

$$
\delta_{\alpha} H=3 \int_{0}^{1}\left(\delta_{\alpha} \eta\right) r^{2} d r
$$

The total time-rate of energy lass of the sample, which is to be identified with tbe observed energy flux, is given by $\ddot{H}_{\text {tot }}=\ddot{H}+\delta_{\alpha} \ddot{H}$. It is important to note that, in making the correction to the calorimetric formulas of section $4, \ddot{H}_{\text {tot }}$ must not be substituted in place of $\dot{H}$. The reason for this is that these formulas were all derived from eq (19) (or its integral equivalent given in eqs (22a) and (23), which in turn was derived from eq (12)), which is a relation between the uncorrected interior enthalpy field and the time-dependence of the surface enthalpy, which is nnaffected by the correction because the boundary condition stated in eq (8a) continues to be valid. Thus the $\mathbf{H}$ that appears in all of the formulas of section 4 must continue to refer to the average value of the uncorrected interior enthalpy field, which means that the right way to make the desired correction is to substitute the righthand side of

$$
\ddot{H}=\ddot{H}_{1 \mathrm{ot}}-\delta_{\alpha} \ddot{H}
$$

wherever $\dot{H}$ appears in a formula, and to identify $\vec{H}_{\text {tot }}$ with the observed heat flux.

The calorimetric formulas of section 4 yield $c(t)$ and $\alpha(t)$ as continuous functions of time from which $c(T)$ and $\alpha(T)$ are found. If, however, spline representations of $c(T)$ and $\alpha(T)$ are used, then the unknowns are the spline coefficients, which are constant numbers. Expressions for the coefficients as weighted integrals involving the observed functions $T(t)$ and $\dot{H}(t)$ could be derived from either the expansion or the integral forms of the calorimetric formulas. Because the spline coefficients are expressed as integrals of the observed data, there would be an automatic smoothing, whïch could be advantageous in the case of noisy data.

Although the calorimetric formulas derived in section 4 were intended to be used with observational data from a single observational run using a single sample, it would also be possible to use them with data from two different runs over the same temperature range using a large and a small sphere of the same material. The radius of the small sphere would be made small enough so that the observations would be in the extreme post transient regime $(T<1 / 4)$ where the accuracy of eq (44) for $c$ would be good. The functional dependence for $c(T)$ found in this way conld then be substituted into the lefthand side of eq (4la), and data from the run with the larger sphere could be inserted into this equation, which would be solved for a. This two-sphere approach had been suggested by Colwell $[1,2]$ when he first proposed radiometric calorimetry of freely cooling spheres.

Because the calorimetric formulas have been derived from an analysis that did not require a knowledge of the cooling law, but rather only the time-dependence of the 
total rate of heat loss (or gain), they could be applied to any situation in which the interior temperature profile of a sphere is determined by the changing temperature of its external environment. For example, in the case of differential scanning calorimetry, using the calorimetric formulas would permit a determination of the thermal parameters of a spherical sample even when the timerate of change of the surface temperature of the sample (i.e., the scanning rate) was so fast that the sample interior would be far from isothermal. This would permit a faster scanning rate, which would in turn cause larger heat fluxes which could be measured with greater precision than the small ones that result when the scanning rate is slow enough to keep the sample interior essentially isothermal. Moreover, the differential scanning technique would no longer be limited to the measurement of heat capacitance, but could also be used for measurements of thermal diffusivity.

Finally, because the analysis does not assume that the measured quantities are monotonic in time, it could be adapted to modulation calorimetry in which the sample surface is subjected to a periodically varying temperature and the magnitude and phase lag of the heat flux as a function of the frequency of the temperature variation are the measured quantities from which the thermal properties are deduced. Although the basic approach of this paper would still be applicable, it would be necessary to subject the surface-driven solution of section 2 to a Fourier analysis in order to express the various quantities as functions of frequency rather than of time.

It was noted in the introduction that the analysis of this paper was the outgrowth of a proposal first made by Dr. J.H. Colwell of the National Bureau of Standards, and throughout the course of this work the author has been the beneficiary of frequent very helpful conversations with Dr. Colwell.

This work was supported by the Materials Processing in Space Division of the National Aeronautics and Space Administration.

\section{References}

[1] Colwell, J.H., NBS: Properties of Electronic Materials, NBSIR 79-1761, J.R. Manning, ed., (National Bnreau of Standards, 1979). Cf. pp. 103-131.

[2] Colwell, J.H., NBS: Materials Measurements, NBSIR 80-2080, J.R. Manning, ed., (National Bureau of Standards, 1980). Cf. pp. 83-113.

[3] Carslaw, H.S.; Jaeger, J.C., Conduction of Heat in Solids, 10xford University Press, New York, 2nd ed., 1959).

[4] Özişik, M.N., Heat Conduction, (John Wiley \& Sons, New York, 1980 ).

[5] Jones, B.F., The Determination of a Coeffieient in a Parabolic Differential Equation Part I. Existence and Uniqueness, J. Math. Mech. 11, 907-918(1962).

[6] Douglas, J.; Jones, B.F., The Delermination of a Coeffieient in a Parabolic Differential Eqnation Part II. Numerical Approximation, J. Math. Mech. 11, 919-926 (1962).

[7] Jones, B.F., Various Methods Ior Finding Unknown Coelficients in Parabolic Differential Equations, Comm. Pure Appl. Math. 16, 33-44 (1963).

[8] Cannon, J.R.; Jones, B.F., Determination of the Diffusivity of an Anisotropic Medium, lnt. J. Eng. Sci. 1, 457-460 (1963).

[9] Cannon, J.R., Determination of Certain Parameters in Heat Conduction Problems, J. Math. Anal. Appl. 8, 188-204 (1964).

[10] Cannon, J.R.; DuChateau, P.C.; Filmer, D.L., A Method of Determining Unknown Rate Constants in a Chemical Reaetion in the Presence of Dilfusion, Math. Bioseiences 9, 61-70 (1970).

[11] Cannon, J.R.; DuChateau, P.C., Determining Unknown Coelfi* eients in a Nonlinear Heat Conduction Problem, SIAM J. Appl. Math. 24, 298-314 (1973).

[12] Cannon, J.R.; DuChateau, P.C., Determination of Unkuown Physical Properties in Hent Conduction Problems, Int. J. Eng. Sci. 11, 783-794 (1973).

[13] Cannon, J.R.; DuChateau, P.C., An Inverse Problem for a Nonlinear Heat Equation, SIAM J. Appl. Math. 39, 272-289 (1980).

[14] Abramowitz, M.; Stegun, I.A., eds., Handbook of Mathematical Functions, Natl. Bur. Staud. (U.S.) Appl. Math. Ser. 55 (1964).

[15] Magnus, W.; Oberhettinger, F.; Soni, R.P., Formulas and Theorems for the Special Functions of Mathematical Physics, (Springer-Verlag, New York, 1966).

[16] Chambré, P.L., Nonlinenr Heat Transler Problem, J. Appl. Phys. 30, 1683-1688 (1959). 